\title{
Duality quantum computer and the efficient quantum simulations
}

\author{
Shi-Jie Wei · Gui-Lu Long
}

July 12,2015

\begin{abstract}
In this paper, we firstly briefly review the duality quantum computer. Distinctly, the generalized quantum gates, the basic evolution operators in a duality quantum computer are no longer unitary, and they can be expressed in terms of linear combinations of unitary operators. All linear bounded operators can be realized in a duality quantum computer, and unitary operators are just the extreme points of the set of generalized quantum gates. A d-slits duality quantum computer can be realized in an ordinary quantum computer with an additional qudit using the duality quantum computing mode. Duality quantum computer provides flexibility and clear physical picture in designing quantum algorithms, serving as a useful bridge between quantum and classical algorithms. In this review, we will show that duality quantum computer can simulate quantum systems more efficiently than ordinary quantum computers by providing descriptions of the recent efficient quantum simulation algorithms of Childs et al [Quantum Information \& Computation, 12(11-12): 901-924 (2012)] for the fast simulation of quantum systems with a sparse Hamiltonian, and the quantum simulation algorithm by Berry et al [Phys. Rev. Lett. 114, 090502 (2015)], which provides exponential improvement in precision for simulating systems with a sparse Hamiltonian.
\end{abstract}

Project supported by the National Natural Science Foundation of China (Grant Nos. 11175094 and 91221205), the National Basic Research Program of China (2011CB9216002).

Shi-Jie Wei · Gui-Lu Long

State Key Laboratory of Low-Dimensional Quantum Physics and Department of Physics, Tsinghua University, Beijing 100084, China

Gui-Lu Long

Tsinghua National Laboratory for Information Science and Technology, Beijing 100084, China

Collaborative Innovation Center of Quantum Matter, Beijing 100084, China

E-mail: gllong@tsinghua.edu.cn 
Keywords Duality computer, duality quantum computer, duality computing mode, quantum divider, quantum combiner, duality parallelism, quantum simulation, linear combination of unitary operators

PACS 03.65.-w, 03.67.-a, 03.75.-b

\section{Introduction}

One of us, Long, came to know Dr. Brandt first through his important works in quantum information $[1,2,3,4,5,6,7,8,9$ and later his role as editor-in-chief of the journal Quantum Information Processing(QIP). Long proposed a new type of quantum computer in 2002 [10, which employed the wave-particle duality principle to quantum information processing. His acquaintance with QIP began in 2006 through the work of Stan Gudder who established the mathematical theory of duality quantum computer [11, which was accompanied by a different mathematical description of duality quantum computer in the density matrix formalism [12. The development of duality quantum computer owes a great deal to QIP first in the term of Dr. David Cory as editor-in-chief, and then the term of Dr. H. E. Brandt as the editor-in-chief. For example, the zero-wave function paradox was pointed out firstly by Gudder [11, and two possible solutions were given in Refs. [13] and 14. Long has actively participated in the work of QIP as a reviewer when Dr. Brandt was the editor-in-chief, and as a member of the editorial board from 2014. At this special occasion, it is our great honor to present a survey of the duality quantum computer in this special issue dedicated to the memory of Dr. Howard E. Brandt.

As is well-known, a moving quantum object passing through a doubleslit behaves like both waves and particles. The duality computer, or duality quantum computer exploits the wave-particle duality of quantum systems 10 . It has been proven that a moving $n$-qubit duality computer passing through a $d$-slits can be perfectly simulated by an ordinary quantum computer with $n$-qubit and an additional $d$ levels qudit $[15,16,17$. So we do not need to build a moving quantum computer device which is very difficult to realize. This also indicates that we can perform duality quantum computing in an ordinary quantum computer, in the so-called duality quantum computing mode [16 17. There have been intensive interests in the theory of duality computer in recent years $[10,11,12,13,14,15,16,17,18,19,20,21,22,23,24,25,26,27,28,29,30$, and experimental studies have also been reported 31,32.

This article is organized as follows. In section 2, we briefly describe the generalized quantum gate, the divider and combiner operations. Section 3 reviews the duality quantum computing mode, which enables the implementation of duality quantum computing in an ordinary quantum computer. In section 4 , we outline the main results of mathematical theory of duality quantum computer. In section 5. we give the duality quantum computer description of the work of Childs et al 33 which simulates a quantum system with sparse Hamiltonian efficiently. In section 6. we give the duality quantum computer description of the work of Berry et al 34] which simulates a quantum system having a sparse 


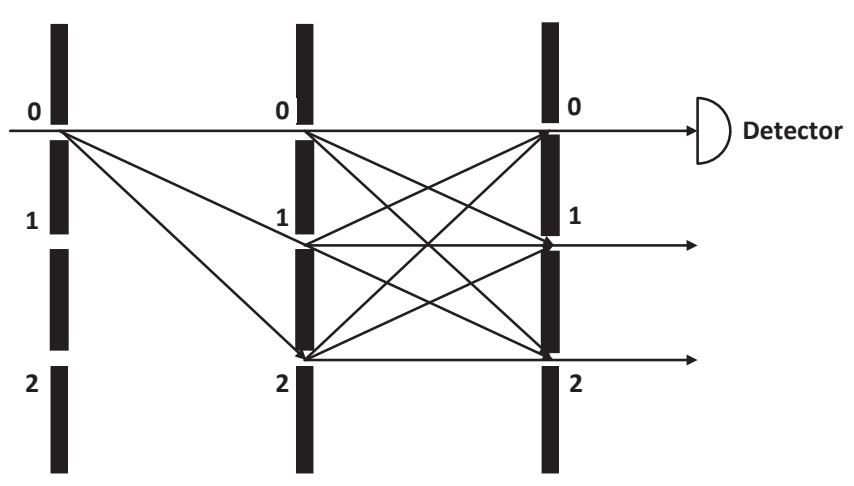

Fig. 1 An illustrated picture for a three-slits duality quantum computer. The input is from the 0 -th slit, and the output of duality quantum computing is taken from only 0th-slit on the right wall [15].

Hamiltonian with exponential improvement in the precision. In section 7 , we give a brief summary.

\section{Duality quantum computer, Divider, Combiner operations}

A duality quantum computer is a moving quantum computer passing through a $d$-slits [10. In Fig. 1, we give an illustration for a duality quantum computer with 3 -slits [15. The quantum wave starts from the 0 -th slit in leftmost wall, and then goes to the middle screen with three slits (this is the divider operation). Between the middle screen and the rightmost screen, some unitary operations are performed on the sub-waves at different slits. They are then collected at the 0 -slit in the rightmost screen, and this is the quantum wave combiner operation. The result is then read-out by the detector placed at the 0 -slit on the right wall.

In a duality quantum computer, the two new operations are the quantum wave divider (QWD) operation and quantum wave combiner (QWC) operation [10. The QWD operation divides the wave function into many identical parts with reduced amplitudes. The physical picture of this division is simple and natural : a quantum system passing through a $d$-slits with its wave function being divided into $d$ sub-waves. Each of the sub-waves has the same internal wave function and are different only in the center of mass motion characterized by the slit. Conversely, the combiner operation adds up all the sub-waves into one wave function. It should be noted that one divides the wave function of the same quantum system into many parts in quantum divider, whereas in quantum cloning one copies the state of one quantum system onto another quantum system [35, 36] (which may also holds true for classical systems 37]). So, the division operation does not violate the non-cloning theorem. 
Considering a quantum wave divider corresponding to a quantum system passing through a $d$-slits. Writing the direct sum of Hilbert space $\oplus_{i=0}^{d-1} H_{i}$ as the form of $H^{\oplus^{d}}$ where $H_{i}=H, i \in\{0, \cdots, d-1\}$. The divider structure characteristics which describes the properties of a quantum wave divider can be denoted as $\left\{p_{i}, i=0, \cdots, d-1\right\}$ where each $p_{i}$ is a complex number with a module less than 1 and satisfy $\sum_{i=1}^{d}\left|p_{i}\right|^{2}=1$. The divider operator $D_{m}$ which maps $H \rightarrow H^{\oplus^{d}}$ is defined by

$$
D_{m} \psi=\oplus_{i=1}^{d-1}\left(p_{i} \psi_{i}\right)
$$

where $\psi_{i}=\psi, i \in\{0, \cdots, d-1\}$. This is the most general form of the divider operator, and it describes a general multi-slits. In a special case, the multi-slits are $d$ identical slits, then $p_{i}=\sqrt{1 / d}$.

The corresponding combiner operation $C_{m}$ can be defined as follows

$$
C_{m}\left(\psi_{0} \oplus \cdots \oplus \psi_{d-1}\right)=\sum_{i=0}^{d-1} q_{i} \psi_{i}
$$

where $q=\left\{q_{0}, \cdots, q_{d-1}\right\}$ denotes the combiner structure that describes the properties of a quantum wave combiner. Each $q_{i}$ is a complex number that satisfy the module less than 1 , and $\sum_{i}\left|q_{i}\right|^{2}=1$. In the case of $q_{i}=\sqrt{1 / d}$, the combiner structure is uniform.

Now, we consider the uniform divider and combiner structures which correspond to $p_{i}=\sqrt{1 / d}$ and $q_{i}=\sqrt{1 / d}$, respectively. In this case, the combined operations of divider and combiner leave the state unchanged. The process can be described as follows

$$
C_{m} D_{m} \psi=C_{m} \oplus_{i=1}^{d} \sqrt{1 / d} \psi_{i}=\sum_{i} 1 / d \psi=\psi
$$

If the divider structure and combiner structure satisfy certain relation, this property also holds. The details will be given in the next section.

It will be shown later in the next section that the divider and combiner structure $D_{m}$ and $C_{m}$ can be expressed by a column or a row of elements of a unitary matrix respectively. For duality quantum gates with the form of $L_{r}$ in Eq.(6), the relation of the two unitary matrices makes the structures of $C_{m}$ and $D_{m}$ adjoint of each other.

\section{Duality Quantum Computing Mode in a Quantum Computer}

The most general form of duality quantum gates has been given in Refs. [16, 17. For the convenience of readers, we use the expressions from duality quantum computing mode 16, 17,23. Compared to ordinary quantum computer where only unitary operators are allowed, the duality quantum computer offers an additional capability in information processing: one can perform different gate operations on the sub-wave functions at different slits [10. This is called 
the duality parallelism, and it enables the duality quantum computer to perform non-unitary gate operations. The generalized quantum gate, or duality gate is defined as follows

$$
L_{c}=\sum_{i=0}^{d-1} c_{i} U_{i}
$$

where $U_{i}$ is unitary and $c_{i}$ is a complex number and satisfies

$$
\sum_{i=0}^{d-1}\left|c_{i}\right| \leq 1
$$

The duality quantum gate is called real duality gate or real generalized quantum gate when it is restricted to positive real $c_{i}$. In this case, $c_{i}$ is denoted by $r_{i}$, and they are constrained by the condition of $\sum_{i} r_{i}=1$. The real duality gate is denoted as $L_{r}$. So, the real duality quantum gate can be rewritten as

$$
L_{r}=\sum_{i=0}^{d-1} r_{i} U_{i}
$$

This corresponds to a physical picture of an asymmetric $d$-slits, and $r_{i}$ is the probability that the duality computer system passes through the $i$-th slit.

According to the definition of duality quantum gates, they are generally non-unitary. It naturally provides the capability to perform non-unitary evolutions. For instance, dynamic evolutions in open quantum systems should be simulated in such machines. More interestingly, it is an important issue to study the computing capabilities of duality quantum computing. An important step toward this direction is that Wang, Du and Dou [19] proposed an theorem which limits what can not be a duality gate in a Hilbert space with infinite degrees of freedom.

The divider operation can be expressed by a general unitary operation $V$ and the combiner operation can be expressed by another general unitary operation $W$. The two unitary operations are implemented on an auxiliary qudit which represents a $d$-slits. The quantum circuit of duality quantum computer is shown in Fig. 2. There are $d$ controlled operations between the operations $V$ and $W$. The $d$ energy levels of the qudit represent the $d$-slits.

We divide the duality computing processing into four steps to reveal the computing theory in a quantum computer.

Step one: Preparing the initial quantum system $|\Psi\rangle|0\rangle$ where $|\Psi\rangle$ is the initial state. Then performing the divider operation by implement the $V$ on the auxiliary qudit, and this operation transforms the initial state into

$$
|\Psi\rangle|0\rangle \rightarrow|\Psi\rangle V|0\rangle=\sum_{i=0}^{d-1} V_{i 0}|\Psi\rangle|i\rangle .
$$

$V_{i 0}$ is the first column element of the unitary matrix $V$ representing the coefficient in each slit. $V_{i 0}=p_{i}$, denotes the divider structure. Note that $V_{i 0}$ 


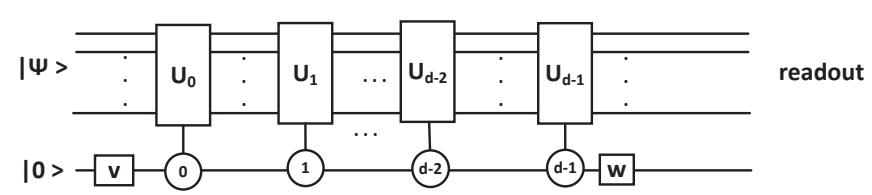

Fig. 2 The quantum circuit of duality computing in a quantum computer. $|\Psi\rangle$ denotes the initial state of duality quantum computer with $|0\rangle$ as the controlling auxiliary qudit. The squares represent unitary operations and the circles represent the state of the controlling qudit, Unitary operations $U_{0}, U_{1} \cdots, U_{d-1}$ are activated only when the qudit holds the respective values indicated in circles [15].

is a complex number with $\left|V_{i 0}\right| \leq 1$ and satisfies $\sum_{i=0}^{d-1}\left|V_{i 0}\right|^{2}=1$. So $V$ is a generalized quantum division operation.

Step two: We perform the qudit controlled operations $U_{0}, U_{1} \cdots, U_{d-1}$ on the target state $|\Psi\rangle$ which leads to the following transformation

$$
\sum_{i=0}^{d-1} V_{i 0} U_{i}|\Psi\rangle|i\rangle .
$$

This corresponds to the physical picture that implements unitary operations simultaneously on the sub-waves at different slits.

Step three: We combine the wave functions by performing the unitary operation $W$. The following state is obtained,

$$
\sum_{i} V_{i 0} U_{i}|\Psi\rangle W|i\rangle
$$

Step four: Detecting the final wave function when the qudit is in state $|0\rangle$ by placing a detector at slit 0 as shown in Fig. 2. The wave function becomes

$$
\sum_{i} W_{0 i} V_{i 0} U_{i}|\Psi\rangle|0\rangle=\sum_{i}\left(W_{0 i} V_{i 0}\right) U_{i}|\Psi\rangle|0\rangle
$$

It should be noted that $W$ is a generalized quantum combiner operation and $q_{i}=W_{0 i}$, which is the combiner structure in Eq. (2). Hence $c_{i}=W_{0 i} V_{i 0}$ is the coefficient in the generalized duality gate defined in Eq. (5). Now, we have successfully realized the duality quantum computing in an ordinary quantum computer.

Considering a special case that $W=V^{\dagger}$, the coefficients $r_{i}=V_{0 i}^{\dagger} V_{i 0}=$ $\left|V_{i 0}\right|^{2}$ satisfy

$$
\sum_{i} r_{i}=\sum_{i}\left|V_{i 0}\right|^{2}=1
$$

where $r_{i}$ is defined in Eq. (6) corresponding to the real duality gate $L_{r}$. 
Generally speaking, $c_{i}=W_{0 i} V_{i 0}$ is a complex number. The sum of $c_{i}$ 's can be denoted as

$$
\sum_{i} c_{i}=\sum_{i} W_{0 i} V_{i 0}=(W V)_{00}
$$

The value of $(W V)_{00}$ is just an element of a unitary matrix $W V$, and naturally has the constraint $\left|(W V)_{00}\right| \leq 1$. Hence the most general form of duality gates allowable by the principles of quantum mechanics is the form of (5)

In a recent study, Zhang et al 22] has proved that it is realizable and necessary to decompose a generalized quantum gate in terms of two unitary operators $V$ and $W$ in Eq. (12) if and only if the coefficients satisfy $\sum_{i}\left|c_{i}\right| \leq 1$. Obtaining the explicit form of the decomposition is a crucial step in duality quantum algorithm design and related studies.

\section{Mathematical theory of duality quantum computer}

The mathematical theory of duality quantum computer has been the subject of many recent studies $[10,11,12,16,17,18,19,20,21,22$. Here we briefly review the mathematical description in duality quantum computing. In this case, the mathematical theory of the divider and combiner operations are restricted to a real structure, namely each $p_{i}(i=0,1, \cdots, d-1)$ is real and positive, and the uniform structure is also a special case of the combiner structure. The following results are from Ref. 11 and we label the corresponding lemma, theorem and corollaries by a letter $\mathrm{G}$, and the corresponding operators are labeled with a subscript $p$. [15].

Here are the properties of generalized quantum gates and related operators

Defining the set of generalized quantum gates on $H$ as $\mathcal{G}(H)$ which turns out to be a convex set. Then we have

Theorem G 4. 1 The identity $I_{H}$ is an extreme point of $\mathcal{G}(H)$, where $I_{H}$ is the identity operator on $H$.

Any unitary operator is in $\mathcal{G}(\mathcal{H})$ and $I_{\mathcal{H}} \in \mathcal{G}(\mathcal{H})$. The identity $I_{H}$ is an extreme point of $\mathcal{G}(H)$ which indicates $\sum_{i} p_{i} U_{i}=I_{\mathcal{H}}$ if and only if $U_{i}=I_{\mathcal{H}}$ for all $i$.

Corollary G 4.2 The extreme points of $\mathcal{G}(\mathcal{H})$ are precisely the unitary operators in $\mathcal{H}$.

We can conclude from Theorem G 4. 1 and Corollary G 4. 2 that the ordinary quantum computer is included in the duality quantum computer.

Denoting $\mathcal{B}(\mathcal{H})$ by the set of bounded linear operators on $\mathcal{H}$ and let $\mathbb{R}^{+} \mathcal{G}(\mathcal{H})$ be the positive cone generated by $\mathcal{G}(\mathcal{H})$. That is

$$
\mathbb{R}^{+} \mathcal{G}(\mathcal{H})=\{\alpha A: A \in \mathcal{G}(\mathcal{H}), \alpha \geq 0\} .
$$

Theorem G 4. 3 If $\operatorname{dim} \mathcal{H}<\infty$, then $\mathcal{B}(\mathcal{H})=\mathbb{R}^{+} \mathcal{G}(\mathcal{H})$.

This theorem shows us that the duality quantum computer is able to simulate any operator in a Hilbert space $\mathcal{H}$ if $\operatorname{dim} \mathcal{H}<\infty$. 
It should be pointed out that these lemmas, corollary and theorems also hold for divider and combiner with a general complex structure [10]. One limitation has been given explicitly by Wang, Du and Dou 19. that what can not be a generalized quantum gate when the dimension is infinite. It is an interesting direction to study the computing ability of duality quantum computer in terms of this theorem.

\section{Description of Childs-Wiebe Algorithm for Simulating Hamiltonians in a Duality Quantum Computer}

Simulating physics with quantum computers is the original motivation of Richard Feynman to propose the idea of quantum computer [38. Benioff has constructed a microscopic quantum mechanical model of computers as represented by Turing machines 39. Quantum simulation is apparently unrealistic using classical computers, but quantum computers are naturally suited to this task. Simulating the time evolution of quantum systems or the dynamics of quantum systems is a major potential application of quantum computers. Quantum computers accelerate the integer factorization problem exponentially through the use of Shor algorithm [40, and the unsorted database search problem in a square-root manner through the Grover's algorithm 41 (see also the improved quantum search algorithms with certainty [42,43]). Quantum computers can simulate quantum systems exponentially fast [38]. Lloyd proposed the original approach to quantum simulation of time-independent local Hamiltonians based on product formulas 44] which attracted many attentions [45, 46, 47, 48, ?]. However, in this formalism, high-order approximations lead to sharply increased algorithmic complexity, the performance of simulation algorithms based on product formulas is limited 33 . For instance, the Lie-TrotterSuzuki formulas, which is high-order product formulas, yields a new efficient approach to approximate the time evolution using a product of unitary operations whose length scales exponentially with the order of the formula 51 . In contrast, classical methods based on multi-product formulas require a sum of unitary operations only in polynomially scales to achieve the same accuracy 52. However, due to the unclosed property of unitary operations under addition, these classical methods cannot be directly implemented on a quantum computer.

The duality quantum computer can be used as a bridge to transform classical algorithms in to quantum computing algorithms. Duality parallelism in the duality quantum computer enables us to perform the non-unitary operations. Moreover,duality quantum gate has the form $L_{c}=\sum_{i=0}^{d-1} c_{i} U_{i}$. This is the linear combinations of unitary operations. Duality quantum computer is naturally suitable for the simulation algorithms of Hamiltonians based on multi-product formulas.

Childs and Wiebe proposed a new approach to simulate Hamiltonian dynamics based on implementing linear combinations of unitary operations 33 . 53. The resulting algorithm has superior performance to existing simulation 
algorithms based on product formulas and is optimal among a large class of methods. Their main results are as follows

Theorem 1 Let the system Hamiltonian be $H=\sum_{j=1}^{m} H_{j}$ where each $H_{j} \in$ $C^{2^{n} \times 2^{n}}$ is Hermitian and satisfies $\left\|H_{j}\right\| \leq h$ for a given constant $h$. Then the Hamiltonian evolution $e^{-i H t}$ can be simulated on a quantum computer with failure probability and error at most $\epsilon$ as a product of linear combinations of unitary operators. In the limit of large $m, h t, 1 / \epsilon$, this simulation uses

$$
\tilde{O}\left(m^{2} h t e^{1.6 \sqrt{\log (m h t / \epsilon)}}\right)
$$

elementary operations and exponentials of the $H_{j} s$.

Considering this simulation algorithm is based on implementing linear combinations of unitary operations, it can be implemented by duality quantum computer. Now, we give the duality quantum computer description of this simulation algorithm.

The evolution operator $U(t)$ satisfies the Schrödinger equation

$$
i \frac{d}{d t} U(t)=H U(t)
$$

and time evolution operator can be formally expressed as $U(t)=e^{-i H t}$.

The Lie-Trotter-Suzuki formulas approximate time evolution operator $U$ for $H=\sum_{j=1}^{m} H_{j}$ as a product of the form

$$
e^{-i H t} \approx\left(\prod_{j=1}^{m} e^{-i H_{j} \frac{t}{r}}\right)^{r}
$$

These formulas can be defined for any integer $\chi>0$ by 33,51 ,

$$
\begin{aligned}
S_{1}(t) & =\prod_{j=1}^{m} e^{-i H_{j} t / 2} \prod_{j=m}^{1} e^{-i H_{j} t / 2} \\
S_{\chi}(t) & =\left(S_{\chi-1}\left(s_{\chi-1} t\right)\right)^{2} S_{\chi-1}\left(\left[1-4 s_{\chi-1}\right] t\right)\left(S_{\chi-1}\left(s_{\chi-1} t\right)\right)^{2},
\end{aligned}
$$

where $s_{\chi-1}=\left(4-4^{1 /(2 \chi-1)}\right)^{-1}$ for any integer $\chi>1$. This choice of $s_{\chi-1}$ is made to ensure that the Taylor series of $S_{\chi}$ matches that of $e^{-i H t}$ to $O\left(t^{2 \chi+1}\right)$. With the values of $\chi$ large enough and the values of $t$ small enough, the approximation of $U(t)$ can reach arbitrary accuracy.

Childs et al have simulated $U(t)$ using $r$ iterations of $M_{k, k}(t / r)$ for some sufficiently large $r$ 33]:

$$
M_{k, k}(t)=\sum_{q=1}^{k+1} C_{q} S_{k}\left(t / \ell_{q}\right)^{\ell_{q}}
$$


where $\ell_{q}(q \in\{1,2, \ldots, k+1\})$ represent distinct natural numbers and $\sum_{q=1}^{k+1} C_{q}=$ $1\left(C_{1}, \ldots, C_{k+1} \in R\right)$. In 33 , the $\ell_{q}$ and $c_{i}$ are defined as

$$
\ell_{q}= \begin{cases}q, & \text { if } q \leq k \\ e^{\gamma(k+1)}, & \text { if } q=k+1\end{cases}
$$

and

$$
C_{q}= \begin{cases}\frac{q^{2}}{q^{2}-e^{2 \gamma(k+1)}} \prod_{j \neq q}^{k} \frac{q^{2}}{q^{2}-j^{2}}, & \text { if } q \leq k, \\ \prod_{j=1}^{k} \frac{e^{2 \gamma(k+1)}}{e^{2 \gamma(k+1)}-j^{2}}, & \text { if } q=k+1 .\end{cases}
$$

The formula is accurate to $O\left(t^{4 k+1}\right)$ order, namely,

$$
\left\|M_{k, k}(\lambda)-U(\lambda)\right\| \in O\left(t^{4 k+1}\right)
$$

The basic idea of this simulation algorithm is that dividing evolution time $t$ into $r$ segments and approximating each time evolution operator segment $U(t / r)=e^{i H t / r}$ by a sum of multi-product formula, namely,

$$
U(t / r) \approx M_{k, k}(t / r)=\sum_{q=1}^{k+1} C_{q} S_{k}\left(t / \ell_{q} r\right)^{\ell_{q}} .
$$

Now, we give a duality quantum computer description of the implementation of this simulation algorithm of time evolution. The quantum circuit is the same as Fig. 2. According to (16), $S_{k}\left(t / \ell_{q} r\right)^{\ell_{q}}$ is an unitary operation. Let $U_{i}=S_{k}\left(t / \ell_{q} r\right)^{\ell_{q}}$ and $c_{i}=C_{q}, M_{k, k}(t / r)$ can be rewritten as

$$
M_{k, k}(t / r)=\sum_{i=0}^{k} c_{i} U_{i}
$$

where $U_{i}$ is an unitary operation.

It is obvious that $M_{k, k}(t / r)$ is a duality quantum gate. The QWD is simulated by the unitary operation $V$ and the QWC is simulates by unitary operation $W$ on a qudit. The auxiliary qudit controlled operations is $U_{i}$. The matrix element $V_{i 0}$ of the unitary matrix $V$ and the matrix element $W_{i 0}$ of the unitary matrix $W$ satisfy:

$$
\begin{aligned}
V_{i, 0} & =\frac{v_{i, 0}}{\sqrt{\sum_{i}\left|v_{i, 0}\right|^{2}}} \\
W_{0, i} & =\frac{w_{0, i}}{\sqrt{\sum_{i}\left|w_{0, i}\right|^{2}}} .
\end{aligned}
$$

As defined in (12), $c_{i}$ is the product of two unitary matrix elements:

$$
c_{i}=W_{0 i} V_{i 0}=\frac{v_{i, 0} w_{0, i}}{\sqrt{\sum_{i}\left|v_{i, 0}\right|^{2}\left|w_{0, i}\right|^{2}}}
$$


The sum of $c_{i}$ 's is

$$
\sum_{i} c_{i}=\sum_{i} W_{0 i} V_{i 0}=(W V)_{00} .
$$

In the special case $W=V^{\dagger}$, the simulation algorithm has the maximum success probability. The expression of $V_{i, 0}$ and $W_{0, i}$ can be simplified into the form:

$$
V_{i, 0}=W_{0, i}=\frac{\sqrt{c_{i}}}{\sqrt{\sum_{i}\left|c_{i}\right|}} .
$$

After implementing the QWD operation, the auxiliary qudit controlled operations and the QWC operation, detecting the final wave function when the auxiliary qudit is in state $|0\rangle$. The initial state $|\Psi\rangle|0\rangle$ has been transformed into

$$
\sum_{i} W_{0 i} V_{i 0} U_{i}|\Psi\rangle|0\rangle=\sum_{i}\left(W_{0 i} V_{i 0}\right) U_{i}|\Psi\rangle|0\rangle=\sum_{i=0}^{k} c_{i} U_{i}|\Psi\rangle|0\rangle .
$$

The approximated evolution operator $M_{k, k}(t / r)$ is implemented successfully by the duality quantum computer. Implementing $r$ segments of $M_{k, k}(t / r)$, we can get the approximation of $U(t)$ by $M_{k, k}(t / r)^{r}$. Thus, this algorithm is clearly realized by the duality quantum computer in straightforward way. The essential idea of this algorithm is an iterated approximation, with each controlled $U_{i}$ adding an additional high order approximation to the evolution operator.

\section{Description of Berry-Childs quantum algorithm with exponential improved precision for a sparse Hamiltonian system}

Berry and Childs provided a quantum algorithm for simulating Hamiltonian dynamics by approximating the truncated Taylor series of the evolution operator on a quantum computer 34. This method is based on linear combinations of unitary operations and it can simulate the time evolution of a class of physical systems. The performance of this algorithm has exponential improvement over previous approaches in precision.

Hamiltonian can be decomposed into a linear combinations of unitary operations:

$$
H=\sum_{\ell=1}^{L} \alpha_{\ell} H_{\ell}
$$

Dividing the evolution time into $r$ segments of length $t / r$. The time evolution operator of each segment can be approximated as

$$
U_{r}:=\exp (-i H t / r) \approx \sum_{k=0}^{K} \frac{(-i H t / r)^{k}}{k !},
$$


where the Taylor series is accurate to order $K$. Substituting the Hamiltonian in terms of a sum of $H_{\ell}$ into (30), we can rewrite the truncated Taylor series as 34 .

$$
U_{r} \approx \widetilde{U}=\sum_{k=0}^{K} \sum_{\ell_{1}, \ldots, \ell_{k}=1}^{L} \frac{(-i t / r)^{k}}{k !} \alpha_{\ell_{1}} \cdots \alpha_{\ell_{k}} H_{\ell_{1}} \cdots H_{\ell_{k}}
$$

For convenience, we can set each $\alpha_{\ell}>0$. Considering $H_{\ell}$ is an unitary operation, we can conclude that the approximation $\widetilde{U}$ is a linear combinations of unitary operations. The expression has a quantum duality gate form. The truncated Taylor series index can be defined as [34]

$$
J:=\left\{\left(k, \ell_{1}, \ldots, \ell_{k}\right) \in J: k \leq K\right\} .
$$

Then, the expression of $\widetilde{U}$ can be simplified as

$$
\widetilde{U}=\sum_{j \in J} \beta_{j} V_{j}
$$

where $\beta_{\left(k, \ell_{1}, \ldots, \ell_{k}\right)}:=\left[(t / r)^{k} / k !\right] \alpha_{\ell_{1}} \cdots \alpha_{\ell_{k}}$ and $V_{\left(k, \ell_{1}, \ldots, \ell_{k}\right)}:=(-i)^{k} H_{\ell_{1}} \cdots H_{\ell_{k}}$. It should be noted that $\widetilde{U}$ is not normalized.

We define the normalization constant as $s=\sum_{j \in J} \beta_{j}$. According to (5), $L_{r}=\widetilde{U} / s$ is a quantum duality gate. We let $r_{i}=\beta_{i} / s, V_{j}=U_{i}$, then it comes back to the duality quantum gate form in (6),

$$
L_{r}=\sum_{i=0}^{d-1} r_{i} U_{i}
$$

where $i \in\{0,1,2, \ldots, d-1\}$.

To give the duality quantum computer description, we need to realize the following processing

$$
|\Psi\rangle|0\rangle \rightarrow|\Psi\rangle \widetilde{U}|0\rangle
$$

In Fig. 3, we give an illustration for our method to perform the algorithm in the form of quantum circuit. The unitary operation $U_{0}$ corresponds to the decomposing form of Hamiltonians: $H=\sum_{\ell=1}^{L} \alpha_{\ell} H_{\ell}$ and the quantum circuit In Fig. 3 implements $U_{r}=\exp (-i H t / r) \approx \sum_{k=0}^{K} \frac{(-i H t / r)^{k}}{k !}$.

The implementation of operation $\widetilde{U}$ need an $L$ level auxiliary qudit $|0\rangle_{L}$ and $K$ auxiliary qubits $|0\rangle^{K}$ which correspond to implementation of two QWD operations and QWC operations. Actually, the equation of (31) indicates that we need summarize twice to realize the right side of this equation. We express the initial state as $|\Psi\rangle|0\rangle^{K}|0\rangle_{L}$ 


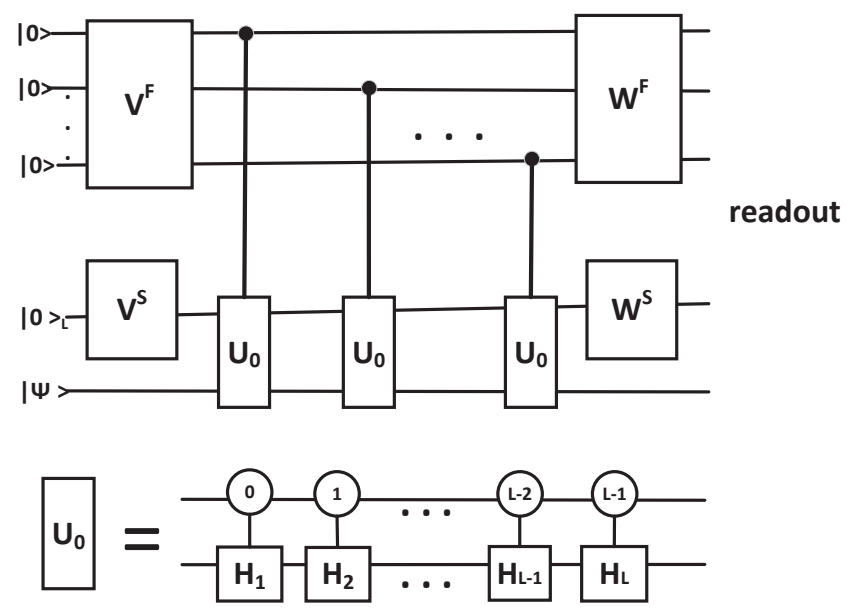

Fig. 3 Duality computing in a quantum computer. $|\Psi\rangle$ is the initial state of duality quantum computer and there are $K$ numbers of $|0\rangle$ auxiliary controlling qubit and an $L$ level auxiliary qudit $|0\rangle_{L}$ auxiliary controlling qudit. Unitary operations $U_{0}$ are activated only when the qubit and qudit holds the respective values indicated in circles. The each unitary operation $U_{0}$ is composed of $H_{1}, H_{2}, \ldots, H_{L-1}, H_{L}$.

Firstly, we transform the $|0\rangle^{K}$ part of the initial state into the normalized state using the QWD operation. We have

$$
|0\rangle^{K} \rightarrow \sum_{k=0}^{K} \sqrt{t^{k} / k !}\left|1^{k} 0^{K-k}\right\rangle .
$$

We let $f=\sum_{0}^{K} \frac{t^{k}}{k !}$ and define the QWD as $V^{F}$, which can be expressed as a $2^{K} \times 2^{K}$ matrix. The elements of the matrix satisfy

$$
V_{i, 0}^{F}=\frac{v_{i, 0}^{F}}{\sqrt{\sum_{i}\left|v_{i, 0}^{F}\right|^{2}}}
$$

where

$$
v_{i, 0}^{F}=\left\{\begin{aligned}
\sqrt{\frac{t^{k}}{k !}}, & i=2^{K}-2^{K-k}, k \in\{0,1, \ldots, K\} . \\
0, & \text { else. }
\end{aligned}\right.
$$

After implementing the first unitary operations $V^{F}$ in the $|0\rangle^{K}$ part of the initial state, we can get normalized state $\frac{\sum_{k=0}^{K} \sqrt{t^{k} / k !}}{\sqrt{f}}\left|1^{k} 0^{K-k}\right\rangle$.

Secondly, using the QWD operation once again to transform the $|0\rangle_{L}$ part of initial state into the normalized state $\sum_{\ell=1}^{L} \sqrt{\alpha_{\ell}}|\ell\rangle$. We let $g=\sum_{\ell=1}^{L} \alpha_{\ell}$ 
and define the second QWD operation as $V^{S}$, which can be expressed as a $L \times L$ matrix. The elements of the matrix satisfy

$$
V_{\ell, 0}^{S}=\frac{v_{\ell, 0}^{S}}{\sqrt{\sum_{\ell}\left|v_{\ell, 0}^{S}\right|^{2}}},
$$

where

$$
v_{\ell, 0}^{S}=\sqrt{\alpha_{\ell}} .
$$

After implementing the second unitary operations $V^{S}$ on $|0\rangle_{L}$ part of initial state, we can get normalized state $\sum_{\ell=1}^{L} \frac{\sqrt{\alpha_{\ell}}}{\sqrt{g}}|\ell\rangle$.

We perform the $L$ level auxiliary qudit $|0\rangle_{L}$ and $K$ auxiliary qubits $|0\rangle^{K}$ controlled operation $U_{i}$ on the computer. The processing can be described as

$$
|\Psi\rangle \rightarrow \sum_{k=0}^{K} \sqrt{t^{k} / k !} \sum_{\ell=1}^{L} \alpha_{\ell} U_{i}|\Psi\rangle
$$

Then, corresponding to the two times of performing of the QWC operations, we need perform the QWC operations twice to combine the wave functions. We define the QWC operations $W^{F}$ and $W^{S}$ corresponding to the QWD operations $V^{F}$ and $V^{S}$ respectively.

We set $W^{F}=\left(V^{F}\right)^{\dagger}, W^{S}=\left(V^{S}\right)^{\dagger}$ and perform the the QWC operations $W^{F}$ and $W^{S}$ on the state $\left|1^{k} 0^{K-k}\right\rangle$ and $|\ell\rangle$, respectively.

After QWC operations $W^{F}$ and $W^{S}$, we detect the final wave function when the auxiliary system is in state $|0\rangle^{K}|0\rangle_{L}$. In the final state, we only focus our attention on the terms with the $L$ level auxiliary qudit in state $|0\rangle_{L}$ and $K$ auxiliary qubits in state $|0\rangle^{K}$. We have the following

$$
\begin{aligned}
& \frac{1}{\sqrt{f}} \sum_{k=0}^{K} \sqrt{t^{k} / k !}\left|1^{k} 0^{K-k}\right\rangle \rightarrow \frac{1}{f} \sum_{k=0}^{K} t^{k} / k !|0\rangle^{K}, \\
& \sum_{\ell=1}^{L} \frac{\sqrt{\alpha_{\ell}}}{\sqrt{g}}|\ell\rangle \rightarrow \frac{1}{g} \sum_{\ell=1}^{L} \alpha_{\ell}|0\rangle_{L} .
\end{aligned}
$$

It should be noted that the summation parts $\sum_{k=0}^{K} \sqrt{t^{k} / k !}$ and $\sum_{\ell=1}^{L} \alpha_{\ell}$ already have been combined with $U_{i}$. The initial state is transformed into

$$
|\Psi\rangle|0\rangle^{K}|0\rangle_{L} \rightarrow \frac{1}{f g} \sum_{k=0}^{K} t^{k} / k ! \sum_{\ell=1}^{L} \alpha_{\ell} U_{i}|\Psi\rangle|0\rangle^{K}|0\rangle_{L},
$$

where $U_{i}$ corresponds to some $(-i)^{k} H_{\ell_{1}} \cdots H_{\ell_{k}}$ and $i \in J$.

It is obvious that $s=f g$ and $\sum_{j \in J} \beta_{j} V_{j}=\sum_{k=0}^{K} t^{k} / k ! \sum_{\ell=1}^{L} \alpha_{\ell}$. Consequently, we have successfully realized the following process: 


$$
|\Psi\rangle|0\rangle^{K}|0\rangle_{L} \rightarrow \frac{1}{s} \widetilde{U}|\Psi\rangle|0\rangle^{K}|0\rangle_{L} .
$$

Finally, the robust form of obvious amplitude amplification procedure of 34] enables us to deterministically implement $\widetilde{U}$ through amplifying the amplitude of $\frac{1}{s} \widetilde{U}$. The approximation accuracy of $\widetilde{U}$ can be quantified by approximation error $\epsilon$. According to the Chernoff bound, as studied in [54], the query complexity is

$$
K=O\left(\frac{\log (r / \epsilon)}{\log \log (r / \epsilon)}\right),
$$

and the error of approximation in each segments satisfy:

$$
\left\|\widetilde{U}-U_{r}\right\| \leq \frac{\epsilon}{r} .
$$

The total number of gates in the simulation for time $t / r$ in each segment is 34

$$
O\left(\frac{L(n+\log L) \log (T / \epsilon)}{\log \log (T / \epsilon)}\right)
$$

In the duality quantum computer description, our method gives a slight improvement than [34, which uses

$$
O\left(\frac{L(n+1) \log (T / \epsilon)}{\log \log (T / \epsilon)}\right)
$$

gates.

Thus, we have given a standard program in the duality quantum computer to realize the simulation methods of Hamiltonians based on linear combinations of unitary operations. The physical picture of our description of the algorithm is clear and simple: each of QWD and QWC operations will lead to one summation of linear combinations of unitary operations. Our method is intuitive and can be easily performed based on the form of time evolution operator.

\section{Summary}

In the present paper, we have briefly reviewed the duality quantum computer. Quantum wave can be divided and recombined by the QWD and QWC operations in a duality quantum computer. The divider and combiner operations are two crucial elements of operations in duality quantum computing and they are realized in a quantum computer by unitary operators. Between the dividing and combining operations, different computing gate operations can be performed at the different sub-wave paths which is called the duality parallelism. It enables us to perform linear combinations of unitary operations in 
the computation, which is called the duality quantum gates or the generalized quantum gates. The duality parallelism may exceed quantum parallelism in quantum computer in the precision aspect.

The duality quantum computer can be perfectly simulated by an ordinary quantum computer with $n$-qubit and an additional qudit, where a qudit labels the slits of the duality quantum computer. It has been shown that the duality quantum computer is able to simulate any linear bounded operator in a Hilbert space, and unitary operators are just the extreme points of the set of generalized quantum gates.

Simulating the time evolution of quantum systems or the dynamics of quantum systems is a major potential application of quantum computers. The property of duality parallelism enables duality quantum computer to simulate the dynamics of quantum systems using linear combinations of unitary operations. It is naturally suitable to realize the simulation algorithms of Hamiltonians based on multi-product formulas which are usually adopted in classical algorithms. The duality quantum computer can be used as a bridge to transform classical algorithms into quantum computing algorithms. We have realized both Childs-Wiebe algorithm and Berry-Childs simulation algorithms in a duality quantum computer. We showed that their algorithm can be described straightforwardly in a duality quantum computer. Our method is simple and has a clearly physical picture. Consequently, it can be more easily realized in experiment 49,50 .

This work was supported by the National Natural Science Foundation of China Grant Nos. (11175094, 91221205), the National Basic Research Program of China (2011CB9216002), the Specialized Research Fund for the Doctoral Program of Education Ministry of China.

\section{References}

1. Brandt, H.E., Myers, J.M., Lomonaco, Jr.S.J.: Aspects of entangled translucent eavesdropping in quantum cryptography. Phys. Rev. A. 56, 4456 (1997)

2. Myers, J.M., Brandt, H.E.: Converting a positive operator-valued measure to a design for a measuring instrument on the laboratory bench. Meas. Sci. Technol. 8, 1222(1997)

3. Brandt, H.E.: Qubit devices and the issue of quantum decoherence. Prog.Quant.Eletron. 22, 257-370 (1999)

4. Brandt, H.E.: Positive operator valued measure in quantum information processing. Am. J. Phys. 67, 434-439 (1999)

5. Brandt, H.E.: Secrecy capacity in the four-state protocol of quantum key distribution. J. Math. Phys. 43, 4526-4530 (2002)

6. Brandt, H.E.: Quantum-cryptographic entangling probe . Phys. Rev. A. 71, 042312 (2005)

7. Brandt, H.E.: Quantum computational geodesics. J. Mod. Opt. 56, 2112-2117 (2009)

8. Brandt, H.E.: Geodesic derivative in quantum circuit complexity analysis. J. Mod. Opt. 57, 1972-1978 (2010)

9. Brandt, H.E.: Aspects of the Riemannian Geometry of Quantum Computation. Int. J. Mod. Phys. B. 26, 1243004 (2012)

10. Long, G.L.: General quantum interference principle and duality computer. Commun. Theor. Phys. 45, 825-844 (2006); Also see arXiv:quant-ph/0512120 It was briefly mentioned in an abstract (5111-53) (Tracking No. FN03-FN02-32) submitted to SPIE conference "Fluctuations and Noise in Photonics and Quantum Optics" in 18 Oct 2002. 
11. Gudder, S.: Mathematical theory of duality quantum computers. Quantum Inf. Process. 6, $37-48$ (2007)

12. Long, G.L.: Mathematical theory of the duality computer in the density matrix formalism. Quantum. Inf. Process. 6(1), 49-54 (2007)

13. Zou, X.F., Qiu, D.W., Wu, L.H., Li, L.J., Li, L.Z.: On mathematical theory of the duality computers. Quantum .Inf. Process. 8, 37-50 (2009)

14. Cui, J.X., Zhou, T., Long, G.L.: Density matrix formalism of duality quantum computer and the solution of zero-wave-function paradox. Quantum. Inf. Process. 11, 317-323 (2012)

15. Long, G.L.: Duality quantum computing and duality quantum information processing Int. J. Theor. Phys. 50, 1305-1318 (2011)

16. Long, G.L., Liu, Y.: Duality computing in quantum computers. Commun. Theor. Phys. 50, 1303-1306 (2008)

17. Long, G.L., Liu, Y., Wang, C.: Allowable generalized quantum gates. Commun. Theor. Phys. 51, 65-67 (2009)

18. Cao, H.X., Li, L., Chen, Z.L., Zhang, Y., Guo, Z.H.: Restricted allowable generalized quantum gates. Chin. Sci. Bull. 55, 2122-2125 (2010)

19. Wang, Y.Q., Du, H.K., Dou, Y.N.: Note on generalized quantum gates and quantum operations. Int. J. Theor. Phys. 47, 2268-2278 (2008)

20. Gudder, S.: Duality quantum computers and quantum operations. Int. J. Theor. Phys 47, 268-279(2008) (also http:// www. math. du. edu /data /preprints/m0611. pdf)

21. Du, H.K., Wang, Y.Q., Xu, J.L.: Applications of the generalized Lders theorem. J. Math. Phys. 49, 013507 (2008)

22. Zhang, Y., Cao, H.X., Li, L.: Realization of allowable qeneralized quantum gates. Sci China-Phys Mech Astron 53, 1878-1883 (2010)

23. Long, G.L., Liu, Y.: Duality quantum computing. Front. Comput. Sci. 2, 167-178 (2008)

24. Long, G.L., Liu, Y.: General principle of quantum interference and the duality quantum computer. Rep.Prog.Phys. 28, 410-431 (2008)(in Chinese)

25. Li, C.Y., Li, J.L.: GENERAL: Allowable Generalized Quantum Gates Using Nonlinear Quantum Optics. Commun. Theor. Phys. 53, 75-77 (2010)

26. Liu, Y., Zhang, W.H, Zhang, C.L., Long, G.L.: Quantum computation with nonlinear optics. Commun. Theor. Phys. 49, 107-110 (2008)

27. Wang, W. Y., Shang, B., Wang, C., Long, G.L.: Prime factorization in the duality computer. Commun. Theor. Phys. 47, 471-473 (2007)

28. Chen, Z.L, Cao, H.X.: A note on the extreme points of positive quantum operations. Int. J. Theor. Phys. 48, 1669-1671 (2010)

29. Hao, L., Liu, D., Long, G.L.: An N/4 fixed-point duality quantum search algorithm. Sci China-Phys Mech Astron 53, 1765-1768 (2010)

30. Liu, Y.: Deleting a marked state in quantum database in a duality computing mode. Chin. Sci. Bull. 58, 2927-2931 (2013)

31. Hao, L., Long, G.L.: Experimental implementation of a fixed-point duality quantum search algorithm in the nuclear magnetic resonance quantum system. Sci China-Phys Mech Astron. 54, 936-941 (2011).

32. Zheng, C., Hao, L., Long, G.L.: Observation of a fast evolution in a parity-timesymmetric system. Philos. Trans. R. Soc. A-Math. Phys. Eng. Sci. 371, 20120053 (2013)

33. Childs, A.M., Wiebe, N.: Hamiltonian simulation using linear combinations of unitary operations. Quantum. Inform. Comput. 12(11-12): 901-924 (2012)

34. Berry, D.W., Childs, A.M., Cleve, R., Kothari, R., Somma, R.D.: Simulating Hamiltonian Dynamics with a Truncated Taylor Series. Phys. Rev. Lett. 114, 090502 (2015)

35. Wootters, W.K., Zurek, W.H.: A single quantum cannot be cloned. Nature, 299, 802-803 (1982)

36. Dieks, D.: Communication by EPR devices. Phys. Lett. A, 92, 271-272 (1982)

37. Yao, S., Liang, H., Gui-Lu, L.: Why can we copy classical information? Chin. Phys. Lett. 28, 010306 (2011)

38. Feynman, R.P.:Simulating physics with computers. Int. J. Theor. Phys. 21, $467(1982)$

39. Benioff, P.: The computer as a physical system: A microscopic quantum mechanical Hamiltonian model of computers as represented by Turing machines. J. Stat. Phys. 22, 563-591 (1980). 
40. Shor, P.W.: Polynomial-time algorithms for prime factorization and discrete logarithms on a quantum computer. SIAM J. Comput. 26, 1484-1509 (1997)

41. Grover, L.K.: Quantum mechanics helps in searching for a needle in a haystack. Phys. Rev. Lett. 79, 325-328 (1997)

42. Long, G.L.: Grover algorithm with zero theoretical failure rate. Phys. Rev. A, 64, 022307 (2001)

43. Toyama, F.M., van Dijk, W., Nogami, Y.: Quantum search with certainty based on modified Grover algorithms: optimum choice of parameters. Quant. Inf. Proc., 12, 18971914 (2013)

44. Lloyd, S.: Universal quantum simulators. Science. 273, 1073 (1996).

45. Lu, Y., Feng, G.R, Li, Y.S, Long, G.L.: Experimental digital quantum simulation of temporalspatial dynamics of interacting fermion system. Sci. Bull. 60, 241-248 (2015)

46. Sornborger, A.T.: Quantum simulation of tunneling in small systems. Sci. Rep. 2, 597 (2012).

47. Childs, A.M., Cleve, R., Deotto, E., Farhi, E., Gutmann, S., Spielman, D.A.: Exponential algorithmic speedup by quantum walk. in Proceedings of the 35th ACM Symposium on Theory of Computing, pp.59-68 (2003)

48. Aharonov, D., Ta-Shma, A.: Adiabatic quantum state generation and statistical zero knowledge. in Proceedings of the 35th ACM Symposium on Theory of Computing, pp. 20-29 (2003)

49. Feng, G.R, Xu, G.F, Long, G.L.: Experimental realization of nonadiabatic holonomic quantum computation. Phys. Rev. Lett. 110,190501 (2013).

50. Feng, G. R., Lu, Y., Hao, L., Zhang, F. H., Long, G. L.: Experimental simulation of quantum tunneling in small systems. Sci. Rep. 3. 2232 (2013)

51. Suzuki, M.: General theory of fractal path integrals with applications to many-body theories and statistical physics. J. Math. Phys. 32, 400 (1991)

52. Blanes, S., Casas, F., Ros, J.: Extrapolation of symplectic integrators.Celest. Mech. Dyn. Astr. 75, 149 (1999)

53. Wiebe, N., Kliuchnikov, V.:Floating point representations in quantum circuit synthesis. New J. Phys. 15, 093041 (2013)

54. Berry, D.W., Childs, A.M., Cleve, R., Kothari, R., Somma, R.D.: in Proceedings of the 46th Annual ACM Symposium on Theory of Computing, New York, 2014(ACM Press, New York, pp. 283292 (2014) 